\title{
Transpiração do tomateiro cultivado fora do solo em estufa plástica e sua relação com os elementos meteorológicos
}

\author{
Transpiration of tomato plants grown outside soil in plastic greenhouse and its relationship with \\ meteorological elements
}

\author{
Josemar Valandro ${ }^{\mathrm{I}}$ Galileo Adeli Buriol ${ }^{\mathrm{II}}$ Jerônimo Luiz Andriolo ${ }^{\mathrm{II}}$ Arno Bernardo Heldwein ${ }^{\mathrm{II}}$
}

\section{RESUMO}

Neste trabalho, foram determinadas as relações da transpiração das plantas do tomateiro (Lycopersicon esculentum M.) cultivado em estufa plástica com os elementos meteorológicos. Realizaram-se três experimentos, no Campo Experimental do Departamento de Fitotecnia da Universidade Federal de Santa Maria - UFSM (RS): no outono-inverno de 1997 e nas primaveras de 1997 e de 1998. Cada planta foi cultivada num volume de aproximadamente oito litros de substrato colocado em sacola plástica. Para a determinação da transpiração diária, foi utilizado um sistema de lisímetros de drenagem, obtendo-se a transpiração pela diferença entre o volume de água irrigado e drenado. A transpiração das plantas foi correlacionada com os elementos meteorológicos radiação solar global diária e média diária da temperatura, umidade relativa e déficit de saturação do ar. Os modelos de regressão obtidos indicaram que é possível estimar a transpiração com razoável acuracidade apenas na primavera. As melhores estimativas foram obtidas com o déficit de saturação do ar em regressão simples $\left(R^{2}=0,814\right)$ e em regressão múltipla com o déficit de saturação do ar e a temperatura do ar $\left(R^{2}=0,881\right)$.

Palavras-chave: Lycopersicon esculentum M., radiação solar, temperatura, déficit de saturação do vapor d’água.

\section{ABSTRACT}

The objective of this research was to establish the relationship between transpiration of the tomato plant (Lycopersycon esculentum M.) grown outside soil in a plastic greenhouse with meteorological elements. The experiment was conducted at the Plant Science Department of the Federal University at Santa Maria and included three experiments: autun-winter 1997, and both spring of 1997 and 1998. The tomato plants were placed in plastic bags with approximately eight liters of fertirrigated susbstrate. In order to measure the daily transpiration, a system of lisymeters was used from were transpiration was measured by substracting irrigated applyed from drained water. Transpiration was correlated with the meteorological elements daily global solar radiation, mean daily temperature, daily mean air humidity and vapor pressure deficit inside the greenhouse. The calculated regression models indicated that is possible to estimate transpiration with reazonable accuracy only during spring season. The best estimates were obtained using vapor pressure deficit with simple regression $\left(R^{2}=0.814\right)$ and temperature and vapor pressure deficit with multiple regression $\left(R^{2}=0.881\right)$.

Keywords: Lycopersicon esculentum, solar radiation, temperature, vapor pressure deficit.

\section{INTRODUÇÃO}

O cultivo do tomateiro em condições naturais no Estado do Rio Grande do Sul, em função de suas exigências térmicas, só é possível nos meses mais quentes do ano. Com a utilização de estufa plástica, o seu cultivo é possível também nos meses mais frios do ano. No interior da estufa, é possível obter, mesmo nos dias mais frios, um acúmulo significativo, com temperaturas acima da temperatura base de crescimento do tomateiro, $10^{\circ} \mathrm{C}$, possibilitando o seu cultivo (MARREROLABRADOR, 1989).

O tomateiro é uma das holerícolas mais cultivadas em estufa plástica. Como conseqüência de seu cultivo sucessivo neste ambiente, a proliferação de alguns patógenos de solo tem sido favorecida. Para contornar este problema, tem-se buscado cultivá-lo fora do solo, utilizando-se substratos artificiais. Esta técnica

IPrograma de Pós-graduação em Agronomia,Universidade Federal de Santa Maria (UFSM), Santa Maria, RS, Brasil.

IIDepartamento de Fitotecnia, UFSM, 97105-900, Santa Maria, RS, Brasil. E-Mail: galileo@unifra.br. Autor para correspondência. 
de cultivo, que teve início na década de 1930 (FAO, 1990), facilita também o manejo da fertirrigação.

No cultivo em substrato artificial, geralmente,o volume de água disponível para as raízes é menor do que no solo e, consequentemente, para manter as plantas permanentemente com suas necessidades hídricas supridas, requer-se um fornecimento de água com mais freqüência.

Já se possuem resultados importantes sobre o consumo de água pelo tomateiro cultivado no interior de estufas plásticas, no solo (DALSASSO, 1997; CARON \& HELDWEIN, 2000). É importante também que seja estudado o consumo de água do tomateiro cultivado no substrato, uma vez que esta técnica, juntamente com a fertirrigação, vem se difundindo na região central do Rio Grande do Sul. Pela insuficiência de conhecimento das necessidades hídricas das plantas conduzidas nesse meio de cultivo, a irrigação ainda é realizada de forma empírica, ocorrendo que ora ela é deficiente, o que acarreta uma redução da fotossíntese pelo fechamento dos estômatos, e ora é excessiva, lixiviando os nutrientes. Dessa forma, é importante quantificar o consumo de água das plantas nesse sistema de cultivo para otimisar e também automatizar o fornecimento de água para elas.

Existe uma estreita relação do consumo de água das plantas cultivadas no interior de estufas com a densidade de fluxo de radiação solar (LAKE et al., 1966; MERMIER et al., 1970; VILLELE, 1972, 1992; BENZARTI et al., 1982). Em função disso, surgiram equações do tipo: $\operatorname{ETP}_{\mathrm{E}}=\mathrm{K} \downarrow$.a $+\mathrm{b}$, em que $\mathrm{ETP}_{\mathrm{E}}$ é a evapotranspiração potencial no interior da estufa, $K \downarrow$ a radiação solar global incidente no interior da estufa e a e b coeficientes da equação de regressão linear. Como no interior da estufa a água do solo se encontra, normalmente, a uma retenção próxima à capacidade de campo, a evapotranspiração, nesse ambiente, é máxima $\left(E_{\mathrm{ETm}}\right)$ desde a implantação de cada cultura e, consequentemente, os valores da razão $\mathrm{ETm}_{\mathrm{E}} / \mathrm{K} \downarrow$ são elevados. Assim, para o tomateiro, MORRIS et al. (1967), STANHILL \& SCHOLTE ALBERS (1974), MUZARD (1976) e STANGHELLINI (1983) encontraram valores de $\mathrm{ETm}_{\mathrm{E}} / \mathrm{K} \downarrow$ que variaram, respectivamente, de 0,6 a 1,0, 0,3 a 0,8, 0,7 a 1,1, e 0,3 a 0,8. A variação deve-se, principalmente, à idade das plantas. Em função desses resultados, VILLLELE (1972) considerou que, para uma cultura de tomateiro com população normal de plantas, a partir do estágio da $4^{\mathrm{a}}$ inflorescência, pode-se tomar 0,8 como valor médio de $\mathrm{ETm}_{\mathrm{E}} / \mathrm{K} \downarrow$.

Baseando-se na dependência do consumo de água pelas plantas da radiação solar incidente no interior da estufa, na França, tem-se estimado a ETP ${ }_{\mathrm{E}}$ ' para uso prático, utilizando-se a equação (VILLELE,
1972): $\operatorname{ETP}_{E}=0,67 \mathrm{~K} \downarrow$, sedo a $\mathrm{ETm}_{\mathrm{E}}$ estimada pelo produto desse valor com o coeficiente de cultura $(\mathrm{Kc})$.

A temperatura do ar no interior da estufa está relacionada, principalmente, com o balanço de energia, dependente, portanto, de fatores tais como a transmissividade do plástico, o ângulo de incidência da radiação solar sobre a cobertura e a renovação do ar do seu interior (MARTINEZ GARCIA, 1978; PRADOS, 1986). A temperatura média diária no interior da estufa é sempre mais elevada que no exterior, sendo que as diferenças variam em torno de 0,5 a $9,0^{\circ} \mathrm{C}$. As maiores diferenças ocorrem das 12 às 16 horas, horários de temperaturas do ar mais elevadas (FARIAS et al., 1993). Já a temperatura mínima do ar no interior é igual ou ligeiramente superior à observada externamente (MONTERO et al., 1985; MILLIS et al., 1990; VILLELE, 1993; BURIOL et al., 1993; HELDWEIN et al., 2001a).

Por sua vez a umidade relativa do ar, durante o período diurno, com o aumento da temperatura, diminui com mais intensidade no interior da estufa do que no ambiente externo, tornando-se geralmente inferior à verificada externamente das 8 às $14 \mathrm{~h}$ (BURIOL et al., 2000). Porém, durante o final da tarde e à noite, aumenta rapidamente. Isto se deve, além de à queda da temperatura verificada neste período no interior da estufa, principalmente, à retenção do ar úmido pela cobertura plástica (TANAKA \& GENTA, 1982; PRADOS, 1986; BURIOL et al., 2000).

O conteúdo de vapor de água do ar no interior da estufa é muito influenciado pela evapotranspiração, pois, em função de se manter o solo e/ou o substrato deste ambiente permanentemente próximos à capacidade de campo por meio de irrigação, a demanda evaporativa do ar é plenamente atendida. Assim, o elevado conteúdo de vapor d'água no ar do interior da estufa origina uma alta pressão parcial de vapor mesmo quando a umidade relativa do ar é baixa (PRADOS,1986; FARIAS et al., 1993; BURIOL et al., 2000). Assim mesmo, os totais de evapotranspiração no interior das estufas são menores do que no exterior, em função, principalmente, da atenuação do fluxo de radiação solar e da velocidade do vento pelo filme plástico. Em uma cultura de feijão-de-vagem, FARIAS et al. (1994) obtiveram uma redução da evapotranspiração de referência (ETo) de 33 a 55\%, segundo os diferentes métodos utilizados no seu cálculo REISSER Jr. (1991), por sua vez, obteve, em cultivo de alface, uma redução da ETm de $27 \%$, nos meses de inverno, e de 33,8\%, nos meses de verão. Com relação à evaporação, BURIOL et al. (2001) constataram que a redução média diária no interior da estufa, em relação ao exterior, em diferentes anos, variou entre 43 e 56\%, quando medida em tanque Classe A, e 
de 37 a $60 \%$ quando medida em evaporímetro de piche. HELDWEIN et al. (2001b), com determinações em minitanques, obtiveram uma redução média da evaporação de $52 \%$ na estufa, em relação ao ambiente externo.

Existe uma relação significativa entre o consumo hídrico do tomateiro, cultivar “Iperor”, de hábito de crescimento determinado, cultivado em estufa plástica, no período do outono, com os elementos meteorológicos, sendo que o déficit de saturação do ar é a variável com que melhor se relaciona (RIGHI, 2000). Para a cultura do pimentão conduzida no interior de estufa, a variável meteorológica que melhor estimou a evapotranspiração máxima também foi o déficit de saturação do ar (DALMAGO, 2001).

Considerando o exposto, constata-se que, nas condições climáticas de Santa Maria, é possível estimar a evapotranspiração do tomateiro cultivado no interior da estufa a partir da variação dos diferentes elementos meteorológicos. Dessa forma, é importante ampliar os estudos das relações do consumo de água do tomateiro com os elementos meteorológicos, utilizando outras cultivares, com distintos hábitos de crescimento.

Com este intuito, no presente trabalho, buscou-se determinar as relações entre a transpiração das plantas de tomateiro, cv. “Monte Carlo”, de hábito indeterminado, cultivadas em substrato no interior de estufa plástica, e a radiação solar, a temperatura do ar e a umidade relativa do ar, em Santa Maria, RS.

\section{MATERIAL E MÉTODOS}

Foram realizados três experimentos, respectivamente no primeiro semestre de 1997, período outono-inverno, e no segundo semestre de 1997 e de 1998, período de primavera, conduzidos no interior de estufa plástica, instalada na Área Experimental do Departamento de Fitotecnia da Universidade Federal de Santa Maria, Santa Maria, RS (latitude: $29^{\circ} 43^{\prime}$ S, longitude: $53^{\circ} 43^{\prime} \mathrm{W}$ e altitude: $95 \mathrm{~m}$ ).

A estufa utilizada foi do tipo capela com cobertura em arco, orientada no sentido norte-sul, medindo 40,00m de comprimento, 10,00m de largura, $2,95 \mathrm{~m}$ de pé direito e $4,50 \mathrm{~m}$ de altura na parte central, coberta com filme de polietileno de baixa densidade (PEBD) transparente.

Foram utilizados os híbridos de tomateiro “Diva”, "Monte Carlo” e "Philipos”, respectivamente para o primeiro, o segundo e o terceiro experimento. As plântulas foram transplantadas num volume de aproximadamente oito litros de substrato, em sacolas plásticas apoiadas sobre camalhões com $0,10 \mathrm{~m}$ de altura e 0,40m de largura. Sobre as sacolas foram estendidos tubos gotejadores para irrigação. Utilizou-se o espaçamento de $0,30 \mathrm{~m}$ entre plantas na fileira e $1,20 \mathrm{~m}$ entre fileiras.

As plantas foram conduzidas verticalmente, em haste única, e os brotos laterais da haste principal foram retirados antes que atingissem $5 \mathrm{~cm}$ de comprimento.

A adubação das plantas foi feita por fertirrigação, por meio do mesmo sistema utilizado para o fornecimento de água, adicionando-se no reservatório de água a solução nutritiva (ANDRIOLO \& POERSCHKE, 1997) sempre que a eletrocondutividade (EC) da solução de drenagem alcançava valores inferiores a $1,0 \mathrm{dS} \mathrm{m}^{-1}$.

A transpiração das plantas foi determinada utilizando-se um lisímetro de drenagem (VALANDRO, et al., 1999). Este consistiu num reservatório de água graduado em $\mathrm{mm}$, com uma capacidade de aproximadamente 32 litros, localizado no centro da estufa, a 1,20m acima do solo e distante 2,40m das plantas. Estas, em número de cinco, foram dispostas a 0,30m uma da outra, cultivadas em substrato colocado dentro de sacolas de plástico, perfuradas na sua base e postas no interior de uma calha de PVC levemente inclinada, para facilitar a drenagem do excesso de água fornecida às plantas. $\mathrm{O}$ fornecimento de água às plantas foi realizado uma vez por dia, sempre nas primeiras horas da manhã. Entretanto, nos meses mais quentes, novembro e dezembro, em função da transpiração ser mais elevada, o fornecimento foi efetuado duas vezes ao dia, em torno das 07 e 13 horas, respectivamente. A transpiração das plantas foi obtida pela diferença entre o volume de água fornecido e o drenado, pois o lisímetro consistiu num sistema em que as perdas de água só ocorriam por transpiração das plantas e por drenagem. A transpiração determinada diariamente foi sempre referente ao dia anterior à determinação. O fornecimento de água para as plantas foi realizado por meio de um sistema constituído de tubos de PVC com diâmetro interno de $1 \mathrm{~mm}$, colocados na base do caule de cada planta, funcionando todos ao mesmo tempo. O controle do volume de água fornecido foi efetuado diretamente com auxílio de uma régua, graduada em $\mathrm{mm}$, fixada na parede externa do reservatório de água, e o volume foi coletado pela drenagem e determinado antes da próxima irrigação, com auxílio de uma proveta graduada em $\mathrm{ml}$. No primeiro experimento, utilizou-se somente um lisímetro e, no segundo e terceiro experimentos, dois e três lisímetros, respectivamente. Em todos os experimentos foram utilizadas cinco plantas em cada lisímetro. As determinações da transpiração das plantas foram diárias, nos períodos compreendidos 
entre 07/05 a 02/07/97, 21/09 a 30/11/97 e 17/08 a 26/11/ 98 , respectivamente, para o primeiro, segundo e terceiro experimentos.

A temperatura e a umidade relativa do ar foram determinadas com auxílio de um termohigrógrafo instalado no interior de um miniabrigo localizado no centro da estufa, a 1,5m da superfície do solo.

A radiação solar global incidente sobre a estufa foi estimada a partir de dados de insolação registrados na estação meteorológica da UFSM, localizada aproximadamente a 100m a sudeste da estufa, utilizando-se as equações apresentadas por ESTEFANEL et al. (1990) para os diferentes meses. Os valores obtidos foram reduzidos em $27 \%$ em função da atenuação da radiação solar pelo filme de polietileno da cobertura da estufa (BURIOL et al., 1995).

O déficit de saturação diário do ar, no interior da estufa, foi obtido pela diferença entre a pressão de saturação de vapor e a pressão parcial de vapor, a partir de valores de temperatura $\left({ }^{\circ} \mathrm{C}\right)$ e umidade relativa do ar (\%).

\section{RESULTADOS E DISCUSSÃO}

Na figura 1a, é apresentada a transpiração das plantas de tomateiro em função da radiação solar global incidente, considerando-se o período de 49 a 105, 2 a 72 e 5 a 106 dias após o transplante, respectivamente, para os experimentos de outonoinverno de 1997 e das primaveras de 1997 e de 1998. A relação entre essas variáveis é positiva, independentemente da época. No experimento de outono-inverno, em função da diminuição da duração do dia e do aumento da inclinação dos raios solares incidentes após o transplante, ocorreu uma diminuição da radiação solar, mesmo as plantas possuindo maior área foliar, massa fresca e seca de folhas e frutos. Já nos experimentos de primavera, o aumento da duração do dia e, conseqüentemente, da densidade de fluxo de radiação solar global ocasionou um contínuo aumento na transpiração das plantas à medida que estas cresceram.

A relação da transpiração do tomateiro com a temperatura do ar (Figura 1b), como ocorre com a radiação solar global, também é positiva. Entretanto, no experimento de outono-inverno, no final do ciclo da cultura, observa-se que, apesar da elevação da temperatura, não houve aumento na transpiração. Isso se deve, principalmente, à baixa incidência de radiação solar nesse período. A elevação da temperatura pode ter sido ocasionada por uma massa de ar quente, que ocasionou a predominância de dias nublados. Conseqüentemente, houve baixa disponibilidade de radiação solar e temperaturas relativamente elevadas, conforme pode ser visto nas figuras $1 \mathrm{a}$ e $1 \mathrm{~b}$, respectivamente. Nesses casos, a estufa permanecia fechada, armazenando grande parte do calor do dia anterior. Nos dias com radiação solar elevada, as cortinas da estufa eram abertas e a temperatura, nessa situação, não se elevava tanto quanto os níveis alcançados quando fechada. Já no período de primavera, a relação entre a transpiração e a temperatura diária foi mais significativa, aumentando ou diminuindo a transpiração segundo a variação da temperatura.

Como ocorre com as variáveis radiação solar global e temperatura do ar, a transpiração das plantas de tomateiro também se relaciona, porém de forma inversa, com a umidade relativa do ar (Figura 1c). Observa-se que, no período de primavera, os valores de umidade relativa do ar foram mais baixos do que no período outono-inverno e, conseqüentemente, o déficit de saturação do ar foi mais elevado (Figura 1d). Isso explica, juntamente com o aumento da radiação solar global e da temperatura do ar, a maior transpiração nesta época.

Na tabela 1, são apresentados os modelos de regressão para estimar a transpiração do tomateiro a partir dos elementos meteorológicos. As variáveis que em modelos de regressão linear simples melhor determinaram a transpiração foram o déficit de saturação do ar (D), no experimento de primavera de $1998\left(\mathrm{R}^{2}=0,814\right)$ e no de outono-inverno de 1997 , embora com coeficiente de determinação bastante baixo $\left(\mathrm{R}^{2}=0,474\right)$ e a temperatura média do $\operatorname{ar}\left(\mathrm{R}^{2}=0,469\right)$, no experimento de primavera de 1987, também com coeficiente de determinação bastante baixo. Em modelos de regressão múltipla, podem ser utilizadas combinações das variáveis $\mathrm{T}+\mathrm{K} \downarrow\left(\mathrm{R}^{2}=0,881\right)$, $\mathrm{D}+\mathrm{T}$ $\left(R^{2}=0,838\right), D+U R\left(R^{2}=0,833\right), D+K \downarrow\left(R^{2}=0,830\right)$ e $T$ + UR $\left(R^{2}=0,808\right)$, no experimento de primavera de 1998 . Já no experimento de outono-inverno de 1997, a combinação das variáveis $\mathrm{D}+\mathrm{K} \downarrow\left(\mathrm{R}^{2}=0,568\right)$ e $\mathrm{K} \downarrow+\mathrm{T}$ $+\mathrm{UR}\left(\mathrm{R}^{2}=0,558\right)$ melhor um pouco a estimativa da transpiração em relação à regressão simples com a variável independente D.

Verifica-se, portanto, que é possível realizar estimativa da transpiração do tomateiro com razoável acurácia apenas na primavera, o que está de acordo com os resultados obtidos por DALSASSO (1997) e CARON (1999) com tomateiro e meloeiro, respectivamente, cultivados no solo. Estes resultados indicam que é possível estimar o consumo de água a partir de variáveis meteorológicas, no período de primavera, para os intervalos de valores que foram desenvolvidos (Tabela 1), sendo porém necessário testar esses modelos antes da sua recomendação. 


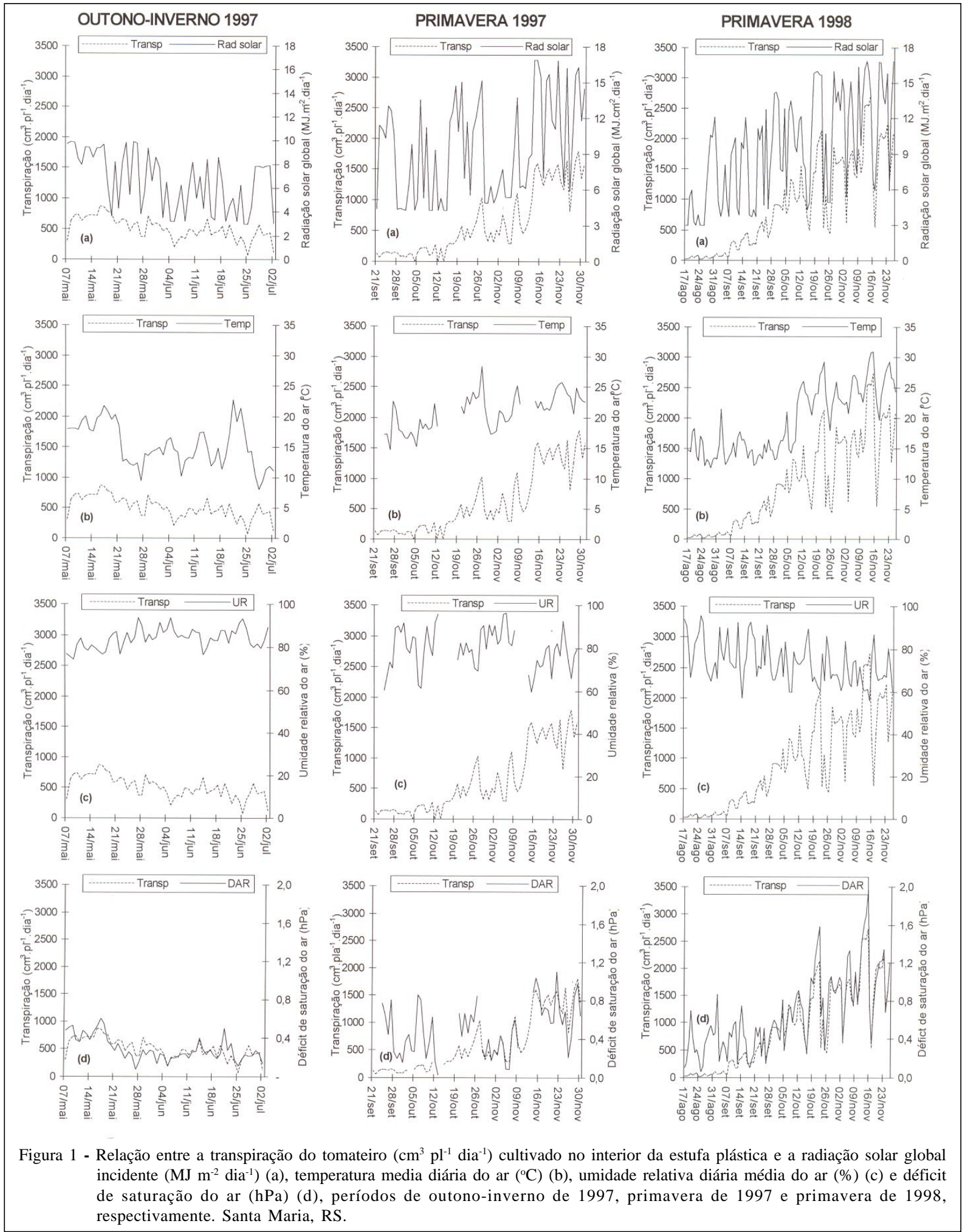

\section{CONCLUSÕES}

Nas condições climáticas de Santa Maria, o consumo de água das plantas de tomateiro foi mais elevado na primavera do que no outono. Foi possível estimar o consumo d'água do tomateiro cultivado em estufa, na primavera, a partir de variáveis meteorológicas. O déficit de saturação do ar foi o 
Tabela 1 - Modelos de regressão para estimar a transpiração do tomateiro cultivado fora do solo em estufa nos períodos outono-inverno de 1997 e primavera de 1997 e de 1998 e conjuntamente 1997/1998, e ainda outono-inverno de 1997 e primavera 1997/1998, a partir dos elementos meteorológicos e respectivos intervalo de validade e coeficiente de determinação ( $\mathrm{R}^{2}$ ). Santa Maria, RS, 1998.

\begin{tabular}{|c|c|c|c|}
\hline $\mathrm{N}$ & Modelo de regressão & Intervalo de validade & $\mathrm{R}^{2}$ \\
\hline \multicolumn{4}{|c|}{ outono-inverno 1997} \\
\hline 57 & $222,063+991,899 \mathrm{D}$ & $3,00<\mathrm{K} \downarrow<9,90$ & 0,474 \\
\hline 57 & $53,701+2177,391 D^{*}-1769,724 D^{2 *}$ & $8,09<\mathrm{T}<22,79$ & 0,494 \\
\hline 57 & $-25,090+45,972 \mathrm{~K} \downarrow+14,969 \mathrm{~T}$ & $74,42<\mathrm{UR}<93,86$ & 0,492 \\
\hline 57 & $128,839+659,937 \mathrm{D}+28,650 \mathrm{~K} \downarrow$ & & 0,568 \\
\hline 57 & $186,237+48,739 \mathrm{~K} \downarrow$ & & 0,434 \\
\hline 57 & 2613,854 - 25,054 UR & & 0,447 \\
\hline 57 & $1583,962+26,815 \mathrm{~K} \downarrow-14,902 \mathrm{UR}$ & & 0,498 \\
\hline 57 & $2260,400+12,432 \mathrm{~T}-23,117 \mathrm{UR}$ & & 0,499 \\
\hline 57 & $1163,104+28,118 \mathrm{~K} \downarrow+13,003 \mathrm{~T}-12,379 \mathrm{UR}$ & & 0,558 \\
\hline \multicolumn{4}{|c|}{ Primavera 1997} \\
\hline 55 & $-1759,911+592,644 \mathrm{D}+19,241 \mathrm{~T}$ & $0,866<\mathrm{D}<1,177$ & 0,529 \\
\hline 55 & $-5385,195+3007,899 \mathrm{D}+56,549 \mathrm{UR}$ & $4,26<\mathrm{K} \downarrow<16,87$ & 0,495 \\
\hline 55 & $-1962,665+126,6187 \mathrm{~T}$ & $14,73<\mathrm{T}<28,40$ & 0,469 \\
\hline 55 & $-597,459+117,5591 \mathrm{~T}-14,8135 \mathrm{UR}$ & $59,60<\mathrm{UR}<96,42$ & 0,523 \\
\hline 55 & $211,204+4,318179 \mathrm{~K} \downarrow^{2}$ & & 0,480 \\
\hline 55 & $-1627,372+84,6899 \mathrm{~T}+56,6814 \mathrm{~K} \downarrow$ & & 0,610 \\
\hline 55 & $-1786,686+115,4541 \mathrm{~K} \downarrow+17,3815$ UR & & 0,486 \\
\hline 55 & $-139,239+87,07516 \mathrm{~K} \downarrow$ & & 0,457 \\
\hline \multicolumn{4}{|c|}{ Primavera 1998} \\
\hline \multirow{2}{*}{102} & $-200,906+1667,346 \mathrm{D}$ & $0,060<\mathrm{D}<1,969$ & 0,814 \\
\hline & $354,727+918,294 \mathrm{D}^{2}$ & $2,93<\mathrm{K} \downarrow<16,87$ & 0,740 \\
\hline 102 & $-338,211+1340,707 \mathrm{D}+36,994 \mathrm{~K} \downarrow$ & $11,79<\mathrm{T}<30,96$ & 0,830 \\
\hline \multirow[t]{2}{*}{102} & $-704,888$ + 1169,156 D + 42,623 T & $56,00<\mathrm{UR}<95,75$ & 0,838 \\
\hline & $-1803,184+2025,230 \mathrm{D}+18,478 \mathrm{UR}$ & & 0,833 \\
\hline 102 & $-1383,361+116,9606 \mathrm{~T}$ & & 0,736 \\
\hline 102 & $-289,399+2,896144 \mathrm{~T}^{2}$ & & 0,757 \\
\hline 102 & $-394,235+136,0124 \mathrm{~K} \downarrow$ & & 0,646 \\
\hline 102 & $120,930+7,092210 \mathrm{~K} \downarrow^{2}$ & & 0,679 \\
\hline 102 & $756,180+97,0276 \mathrm{~T}-23,6966 \mathrm{UR}$ & & 0,808 \\
\hline 102 & $-1422,087+80,4904 \mathrm{~T}+78,5426 \mathrm{~K} \downarrow$ & & 0,881 \\
\hline \multicolumn{4}{|c|}{ Primavera 1997 e 1998} \\
\hline \multirow[t]{2}{*}{157} & $-131,973+1560,179 D^{*}$ & $0,060<\mathrm{D}<1,969$ & 0,693 \\
\hline & $363,990+910,510 \mathrm{D}^{2}$ & $2,9<\mathrm{K} \downarrow<16,87$ & 0,641 \\
\hline 157 & $-333,139+1132,530 \mathrm{D}+48,555 \mathrm{~K} \downarrow$ & $11,79<\mathrm{T}<30,96$ & 0,734 \\
\hline 157 & $-876,077+1041,457 \mathrm{D}+53,013 \mathrm{~T}$ & $56,00<\mathrm{UR}<96,40$ & 0,751 \\
\hline 157 & $-1109.914+578,292 \mathrm{D}+54,705 \mathrm{~T}+50,708 \mathrm{~K} \downarrow$ & & 0,797 \\
\hline 157 & $-1435,229+112,9890 \mathrm{~T}$ & & 0,619 \\
\hline 157 & $1277,772-170,0467 \mathrm{~T}+6,972933 \mathrm{~T}^{2}$ & & 0,688 \\
\hline 157 & $-376,3064+2,8100 \mathrm{~T}^{2}$ & & 0,653 \\
\hline 157 & $-311,909+119,5565 \mathrm{~K} \downarrow$ & & 0,573 \\
\hline 157 & $-1413,5994+73,9382 \mathrm{~K} \downarrow+76,5635 \mathrm{~T}$ & & 0,774 \\
\hline \multicolumn{4}{|c|}{ Outono-inverno 1997 - Primavera 1997 e 1998} \\
\hline \multirow[t]{2}{*}{214} & $-18,231+1442,11509 \mathrm{D}$ & $0,060<\mathrm{D}<1,969$ & 0,688 \\
\hline & $383,325+898,893018 \mathrm{D}^{2}$ & $2,93<\mathrm{K} \downarrow<16,87$ & 0,655 \\
\hline 214 & $76,660+1074,460 \mathrm{D}+247,505 \mathrm{D}^{2} *$ & $3,00<\mathrm{T}<30,96$ & 0,692 \\
\hline 214 & $-234,134+1327,206 \mathrm{D}+24,871 \mathrm{~K} \downarrow$ & $56,00<\mathrm{UR}<96,42$ & 0,722 \\
\hline 214 & $-757,945+666,754 \mathrm{D}+35,401 \mathrm{~T}+50,988 \mathrm{~K} \downarrow$ & & 0,768 \\
\hline 214 & $-2233,73+2024,2330 \mathrm{D}+24,3500 \mathrm{UR}$ & & 0,739 \\
\hline 214 & $140,957+1,864 \quad \mathrm{~T}^{2}$ & & 0,519 \\
\hline 214 & $972,650-125,4060 \mathrm{~T}+5,656607 \mathrm{~T}^{2}$ & & 0,629 \\
\hline 214 & $2112,778+35,539 \mathrm{~T}-25,035 \mathrm{UR}$ & & 0,504 \\
\hline 214 & $-1100,391+61,657 \mathrm{~T}+74,6840 \mathrm{~K} \downarrow$ & & 0,734 \\
\hline 214 & $2958,280+34,601 \mathrm{~K} \downarrow-33,306$ UR & & 0,437 \\
\hline
\end{tabular}

$\mathrm{N}$ = número de observações; * = significativo a 5\%, sendo as demais variáveis significativas a $1 \%$ de probabilidade; Trans = transpiração de água por planta $\left(\mathrm{cm}^{3} \cdot \mathrm{pl}^{-1} \cdot \mathrm{dia}^{-1}\right) ; \mathrm{K} \downarrow=$ radiação solar global diária $\left(\mathrm{MJ}^{-\mathrm{m}^{-2}} \cdot \mathrm{dia}^{-1}\right)$; $\mathrm{T}=$ temperatura média diária do ar $\left({ }^{\circ} \mathrm{C}\right)$; UR $=$ umidade relativa média diária do ar (\%) e $\mathrm{D}$ = déficit de saturação do ar (hPa). 
elemento meteorológico que mais influenciou o consumo de água do tomateiro cultivado em estufa plástica, tanto na primavera quanto no outono.

\section{REFERÊNCIAS}

ANDRIOLO, J.L.; POERSCHKE, P.R.C. Cultivo do tomateiro em substratos. Santa Maria: UFSM - Centro de Ciências Rurais, 1997. 12p. (Informe Técnico, 2)

BENZARTI, J. et al. Etude expérimentale de l'évapotranspiration potentialle sous serre en climat semmi aride. Annales INRA Tunisie, Ariona, v.55, n.1, p.1-24, 1982.

BURIOL, G.A. et al. Modificação na temperatura mínima do ar causada por estufas de polietileno transparente de baixa densidade. Revista Brasileira de Agrometeorologia, Santa Maria, v.1, n.1, p.43-49, 1993.

BURIOL, G.A. et al. Transmissividade à radiação solar do polietileno de baixa densidade utilizado em estufas. Ciência Rural, Santa Maria, v.25, n.1, p.1-4, 1995.

BURIOL, G.A. et al. Modificação da umidade relativa do ar pelo uso e manejo da estufa plástica. Revista Brasileira de Agrometeorologia, Santa Maria, v.8, n.1, p.11-18, 2000.

BURIOL, G.A. et al. Evaporação d’água em estufas plásticas e sua relação com o ambiente externo: 1 - avaliação com o uso do tanque classe A e o evaporímetro de Piche. Revista Brasileira de Agrometeorologia, Santa Maria, v.9, n.1, p.35-41, 2001.

CARON, B.O. Consumo d'água e coeficiente de cultura do meloeiro cultivado em estufa plástica. 1999. 71f. Dissertação (Mestrado em Agronomia) - Universidade Federal de Santa Maria.

CARON, B.O.; HELDWEIN, A.B. Consumo d’água e coeficiente de cultura para o meloeiro cultivado em estufa plástica na primavera. Revista Brasileira de Agrometeorologia, Santa Maria, v.8, n.1, p.19-25, 2000.

DALMAGO, G.A. Evapotranspiração máxima e sua modelagem para a cultura do pimentão em estufa plástica. 2001. 166f. Dissertação (Mestrado em Agronomia) - Universidade Federal de Santa Maria.

DALSASSO, L.C.M. Consumo d'água e coeficiente de cultura do tomateiro (Licopersicum esculentum, M.) e do pepino (Cucumis sativus, $L$.) cultivados em estufa plástica. 1997. 84f. Dissertação (Mestrado em Agronomia) Universidade Federal de Santa Maria .

ESTEFANEL, V. et al. Insolação e radiação solar de Santa Maria, RS: I - Estimativa da radiação solar global incidente a partir dos dados de insolação. Revista do Centro de Ciências Rurais, Santa Maria, v.20, n.3-4, p.303-218, 1990.

FAO. Soilles culture for horticultural crop production. Rome: Food and Agriculture Organization of the United Nations,
1990. 188p. (FAO- Plant Production and Protection Paper, 101).

FARIAS, J.R.B. et al. Alterações na temperatura e umidade relativa do ar provocados pelo uso de estufa plástica. Revista Brasileira de Agrometeorologia, Santa Maria, v.1, n.1, p.5162, 1993.

FARIAS, J.R.B. et al. Evapotranspiração no interior de estufas plásticas. Revista Brasileira de Agrometeorologia, Santa Maria, v.2, p.17-22, 1994.

HELDWEIN, A.B. et al. Variação vertical de temperatura do ar no interior de estufas plásticas. Revista Argentina de Agrometeorologia, Buenos Aires, v.1, n.1, p.35-42, 2001a.

HELDWEIN, A.B. et al. Evaporação d’água em estufas plásticas e sua relação com o ambiente externo; 2 - efeito da espécie cultivada e da época do ano nos valores obtidos com minitanques. Revista Brasileira de Agrometeorologia, Santa Maria, v.9, n.1, p.43-49, 2001b.

LAKE, J.V. et al. Seasonal variation in the transpiration of glasshous plants. Agricultural Meteorology, Amsterdan, v.3, p.183-196, 1966.

MARRERO LABRADOR, P. Day degress requisedad for growth at gome station in la Habana Province. Horticulturae Abstracts, East Malling, v.59, n.9, p.862, 1989.

MARTINEZ GARCIA, P.F. Caracteristicas climáticas de los invernadores de plástico. Madrid: Instituto Nacional de investigaciones Agrárias - INIA, 1978. 48p.(Hojas Tecnicas,19).

MERMIER, M. et al. Premières mesures d'évaporation sous serre. Pépiniéristes Horticulteurs Maroîchers, Paris, v.103, p.6255-6260, 1970 .

MILLIS, P.J.W.; et al. A greenhouse design for a cool subtropical climate with mild winters based in microclimatic mesuraments of protected environments. Acta Horticulturae, Wageningen, n.281, p.83-93, 1990.

MONTERO, J.I. et al. Climate under plastic in the Almeria area. Acta Horticulturae, Wageningen, n.17, p.227-234 1985.

MORRIS, L.G. et al. The transpiration of glasshouse crops and its relationships to the incoming solar radiation. Journal of Agricultural Engnineering Research, Silsoe, v.2, n.2, p.11122, 1967

MUZARD, M. Besoins en eau des cultures maraîchères sous serre et conduit de l'irrigation en fonction du rayonnement solaire global. Pépiniérists Horticulteurs Moraîchers, Paris, v.165, p.23-31, 1976.

PRADOS, N.C. Contribución al estudio de los cultivos enarenados en Almeria: necessidades hídricas y extración del nutrientes del cultivo de tomate de crescimneto indeterminado en abrigo de polietileno. 1986. 195f. Tesis (Doutorado em Fitotecnia) - Caja Rural Principal, Almeria.

REISSER Jr., C. Evapotranspiração da alface em estufa plástica e ambiente natural. 1991. 78f. Dissertação 
(Mestrado em Irrigação e Drenagem) - Curso de Pós-graduação em Engenharia Agrícola, Universidade Federal de Santa Maria.

RIGHI, E.Z. Consumo hídrico do tomateiro (Licopersicon esculentum Mill.) cultivado em estufa plástica e sua relação com variáveis meteorológicas em Santa Maria. 2000. 83f. Dissertação (Mestrado em Agrometeorologia) Escola Superior de Agricultura Luiz de Queiroz/USP.

STANGHLLINI, C. Evaporation of a greenhouse crop and its relationship to the supply of heat. Wageningen: IMAG, 1983. 31p.

STANHILL, G.; SCHOLTE ALBERS, J. Solar radiation and water loss from glasshouse roses. Journal of the Americam Societis Horticultural Science, Mount Vernon, v.99, n.2, p.107-110, 1974.
TANAKA, M.; GENTA, H. Control del medio ambiente baja invernadero y tunel plastico. Salto: Estacion Experimental de Citricultura, 1982. 61p.

VALANDRO, J. et al. Dispositivo Lisimétrico simples para determinar a transpiração das hortaliças cultivadas fora do solo. Revista Brasileira de Agrometeorologia, Santa Maria, v.7, n.2, p.189-193, 1999.

VILLELE, O. de. Les besoins en eau des cultures serre. Essai de conduite des arrosages en function de l'ensoleillement. Acta Horticulturae. Den Haag, v.35, p.123-130, 1972.

VILLELE, O. de. Les besoins en eau des cultures. Montfavet: INRA, 1992. 16p.

VILLELE, O. de. Le contexte climatic et culture de la serre. 1 - La serre, agent de modificacion du climat. In: L'INRA et les cultures sous serre. Paris: INRA, 1993. p.21-27. 